\title{
Elastic Properties of Chemically Derived Single Graphene Sheets
}

2008

Vol. 8, No. 7 2045-2049

\author{
Cristina Gómez-Navarro, ${ }^{\star, \dagger}$ Marko Burghard, ${ }^{\dagger}$ and Klaus Kern ${ }^{\dagger, \neq}$ \\ Max-Planck-Institut für Festkörperforschung, Heisenbergstrasse 1, \\ 70569 Stuttgart, Germany, and Ecole Polytechnique Federale de Lausanne, \\ CH-1015 Lausanne, Switzerland
}

Received May 14, 2008

\begin{abstract}
The elastic modulus of freely suspended graphene monolayers, obtained via chemical reduction of graphene oxide, was determined through tip-induced deformation experiments. Despite their defect content, the single sheets exhibit an extraordinary stiffness $(E=0.25 \mathrm{TPa})$ approaching that of pristine graphene, as well as a high flexibility which enables them to bend easily in their elastic regime. Built-in tensions are found to be significantly lower compared to mechanically exfoliated graphene. The high resilience of the sheets is demonstrated by their unaltered electrical conductivity after multiple deformations. The electrical conductivity of the sheets scales inversely with the elastic modulus, pointing toward a 2-fold role of the oxygen bridges, that is, to impart a bond reinforcement while at the same time impeding the charge transport.
\end{abstract}

Recent experiments have revealed the thermodynamic stability of graphene under ambient conditions, ${ }^{1-3}$ which strongly revived the interest in the electrical and mechanical properties of this fascinating carbon nanostructure. Two major methods have been established for the fabrication of graphene monolayers, namely, mechanical exfoliation of graphite ${ }^{2}$ and vacuum graphitization of silicon carbide. ${ }^{3,4}$ More recently, chemical reduction of graphene oxide (GO) has been reported as an alternative, solution-based route, for obtaining graphenelike sheets which offers the advantages of being cheap and up-scalable..$^{5-8}$ Even though GO is a good insulator, deoxygenation has been shown to substantially enhance its electrical conductivity, albeit the obtained values of $\sim 1 \mathrm{~S} / \mathrm{cm}$ remain $2-3$ orders of magnitude below that of pristine graphene. ${ }^{6,7}$ Due to the limited efficiency of the reduction process, the obtained sheets still contain residual oxygenated functional groups of the starting material. Microscopic studies of the reduced GO also indicate the coexistence of graphitic regions with defect clusters ${ }^{6,9}$ in agreement with proposed models. ${ }^{9-11}$ In addition to its interesting electrical characteristics, this $2 \mathrm{D}$ material is expected to have also unique mechanical properties. Recent studies have witnessed its suitability for the fabrication of composites ${ }^{12,13}$ and paperlike materials ${ }^{14}$ with excellent mechanical properties. However, in order to promote its application in nanotechnology, the mechanical characterization of single sheets is of upmost relevance.

\footnotetext{
* Corresponding author, c.gomez-navarro@fkf.mpg.de.

$\dagger$ Max-Planck-Institut für Festkörperforschung.

¥ Ecole Polytechnique Federale de Lausanne.
}
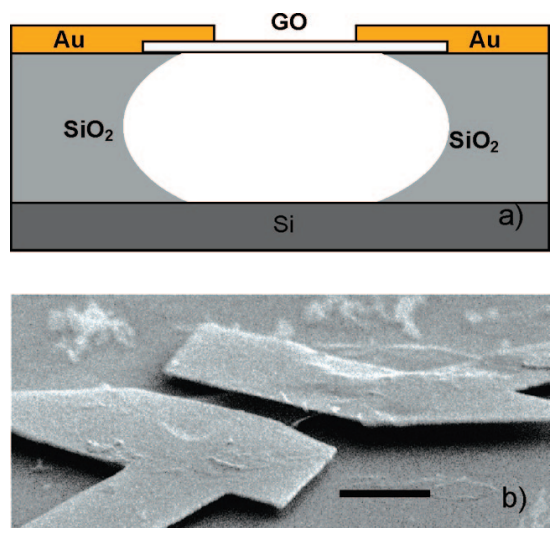

Figure 1. Device geometry: (a) Schematic depiction of the sample geometry (lateral view, not to scale). The highly doped Si wafer serves as a back gate in the electrical measurements. (b) Scanning electron microscopy image of a suspended reduced graphene oxide monolayer, for which the electrode undercut can be clearly discerned (scale bar corresponds to $1 \mu \mathrm{m}$ ).

Here we present a study of the mechanical and electrical properties of suspended, chemically reduced GO single sheets. Graphite oxide prepared via Hummers method ${ }^{15}$ was dispersed in water, deposited onto a $\mathrm{Si} / \mathrm{SiO}_{2}$ substrate, and subsequently reduced by hydrogen plasma treatment. The obtained samples exhibited a high yield of monolayers with lateral dimensions of $0.1-5 \mu \mathrm{m}$. Preselected layers were then contacted by standard e-beam lithography with $\mathrm{Ti} / \mathrm{Au}$ electrodes. In order to freely suspend the layers (Figure 1), the samples were wet etched by buffered hydrofluoric acid, followed by critical point drying to prevent adhesion of the sheets to the substrate. Figure $2 \mathrm{a}, \mathrm{b}$ displays two atomic force 

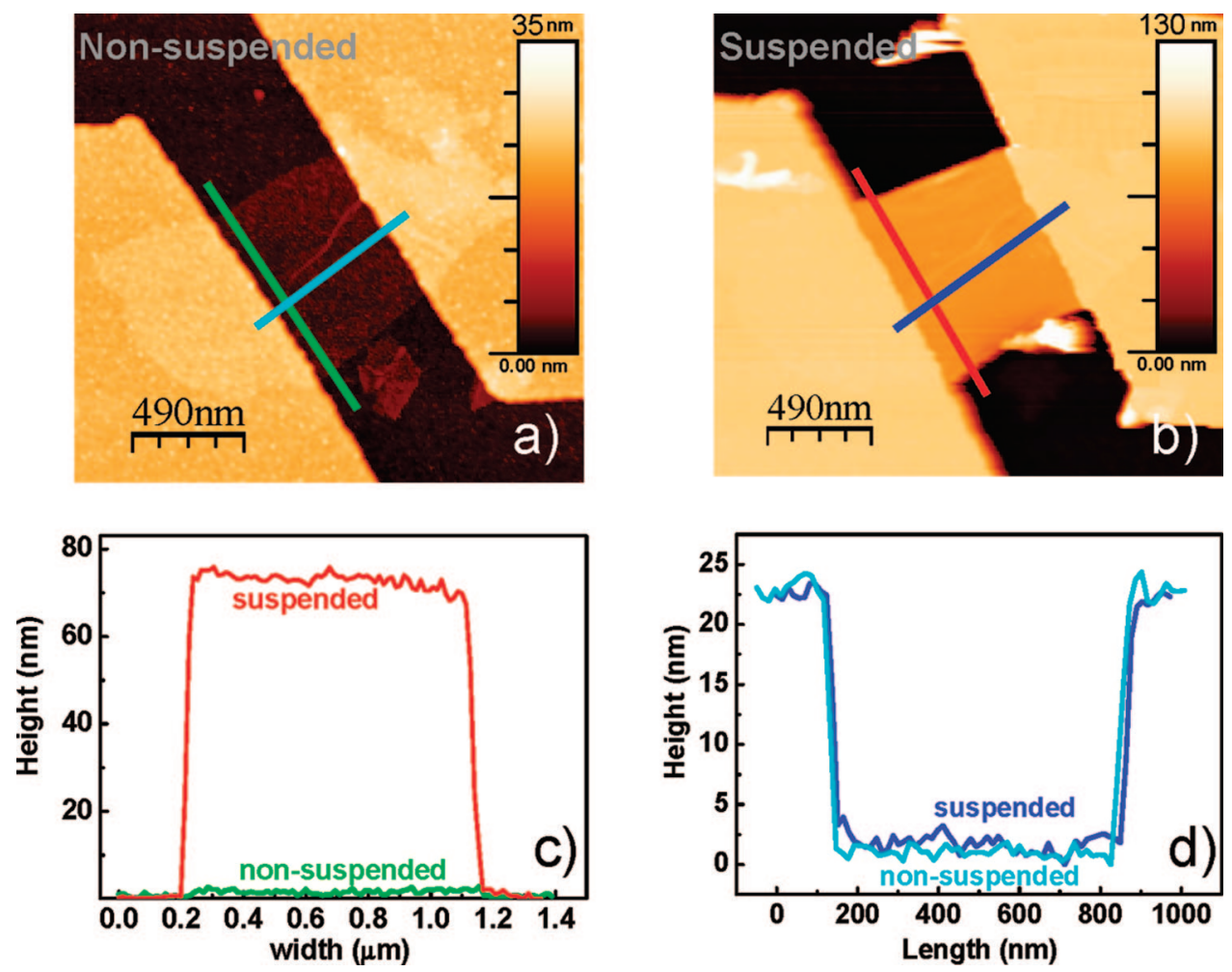

Figure 2. Atomic force microscopy images of a reduced GO monolayer before and after etching. The AFM topographic images show the same GO sheet before (a) and after (b) rendering it free-standing. The color scales are different for the two images. Panels c and d display AFM height profiles along the lines marked in color in the AFM images. The profiles indicate a channel depth of $\sim 75 \mathrm{~nm}(\mathrm{c})$, and the absence of slack along the length of the suspended layer (d).

microscopy (AFM) topographic images of the same GO monolayer before and after etching. AFM height profiles of the etched sample taken along the width and length of the channel (Figure 2c,d) confirm the presence of a free-standing structure. From these profiles, a channel depth of $75-80 \mathrm{~nm}$ is determined. Due to the different etching rates in the lateral and vertical directions, this depth is sufficient to ensure complete suspension of the layer. In the present work, we were able to realize suspended GO sheets with sizes of up to $1 \mu \mathrm{m}^{2}$. Larger sheets were found to collapse onto the bottom of the trenches most likely due to residual tensions surface during the drying process. A few percent of the smaller layers appeared to be buckled toward the substrate. For the experiments described in the following, only fully suspended monolayers with no observable slack were selected.

Mechanical characterization of the free-standing reduced GO monolayers was performed by indenting an AFM tip at the center of the suspended area. Before indentation, the sheets were localized by AFM imaging in noncontact dynamic mode in order to avoid damage of the samples. Four different force vs $z$ piezo displacement curves are displayed in Figure 3a. Curves acquired on the $\mathrm{Si} / \mathrm{SiO}_{2}$ substrate were used as a reference for calculating the applied force $(F)$ and the resulting deflection of the layers $(\delta)$. The obtained $F(\delta)$ curves are linear for small deflections but assume a nonlinear dependence for deflections exceeding $\sim 10 \mathrm{~nm}$. The transition to a nonlinear regime is predicted by elasticity theory and can be ascribed to the onset of sizable local deformations originating from the finite size and shape of the indenter. ${ }^{16-18}$
Importantly, even for large deflections of $50 \mathrm{~nm}$ (corresponding to strains of $\sim 1 \%$ ), the loading and unloading curves were observed to overlap, indicating fully elastic deformations under these conditions. Further evidence for the excellent resilience of the sheets derives from the fact that the slope of the $F(\delta)$ curves does not change after repeated deformations, which proves the absence of permanent damage. Dynamic complications such as viscosity effects can be ruled out since the curves do not depend on the approaching or retracting speed of the tip (in the range $10-500 \mathrm{~nm} / \mathrm{s})$.

The $F(\delta)$ curves provide access to the stiffness ( $\left.K_{\text {eff }}\right)$ of the GO sheets. According to continuum mechanics, the effective force constant of a double-clamped beam depends on both the mechanical properties of the material and the geometry of the beam. In the pure bending regime (i.e., small indentations) and for point loading at the beam center, $K_{\text {eff }}$ is related to the elastic modulus, $E$, of the material by the expression

$$
K_{\mathrm{eff}}=32 E w(t / l)^{3}+17 T / l
$$

where $t, w$, and $l$ are the thickness, width, and length of the beam, respectively, and $T$ is the tension in the beam. In the limit of $T \rightarrow 0$, a linear scaling of $K_{\text {eff }}$ with $w(t / l)^{3}$ would thus be expected. By contrast, the corresponding plot of the experimental data of $K_{\text {eff }}$ (Figure 4a) exhibits a pronounced scatter, indicating the presence of sizable built-in tensions $(T)$. The magnitude of this tension can be derived from the measured $K_{\text {eff }}$ values and the sheet dimensions: eq 1 can be rewritten as $E=32(l / t)^{3} K_{\text {eff }} / w-17 T l^{2} / 32 w t^{3}$ where the first 

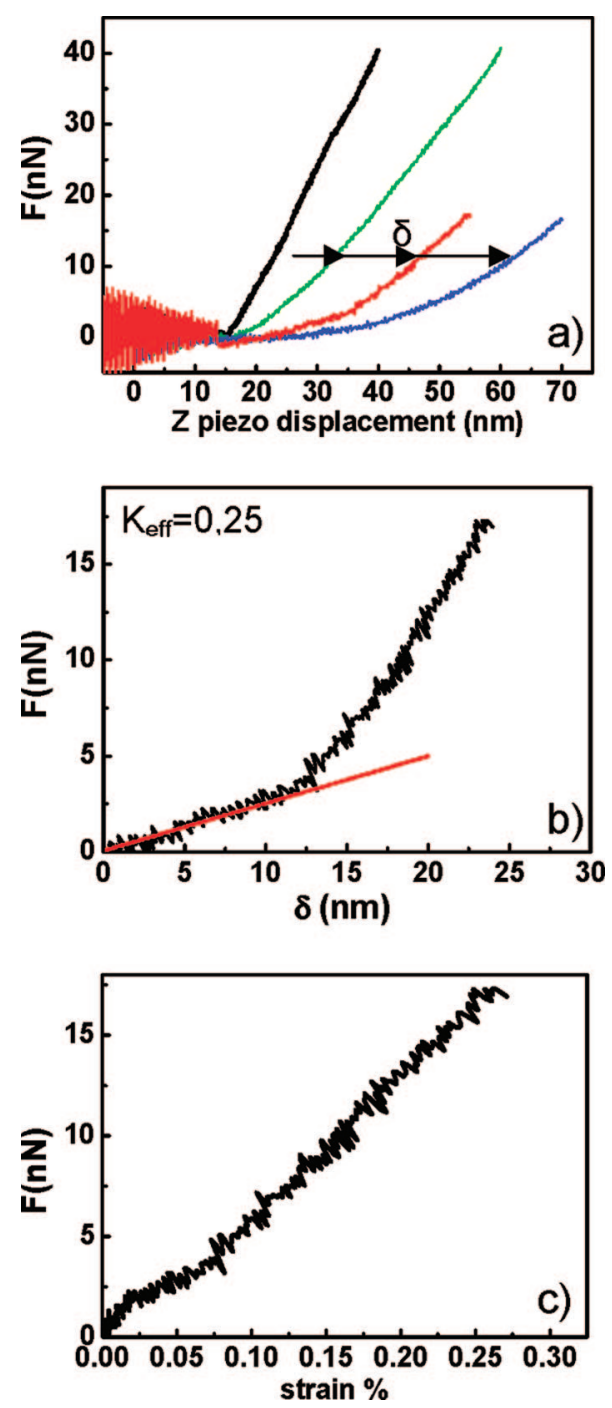

Figure 3. Deformation response of suspended GO monolayers. (a) Plot of force as a function of vertical piezo displacement of the AFM scanner for a hard substrate (black curve) and three different suspended layers (colored curves) with different geometries. The deformation depth is calculated as the difference in $Z$ for a fixed force. (b) Force vs deformation depth curve corresponding to the red curve in panel a. The dashed red line indicates the linear dependence for small deformations, which is the basis for the calculation of the elastic modulus. (c) Force vs strain curve extracted from the plot in panel $b$. The strain has been calculated as the relative change in length, assuming a point load and a triangular strained geometry.

term is obtainable from the experimental data according to $E_{\text {eff }}=32(l / t)^{3} K_{\text {eff }} / w$. This effective modulus $\left(E_{\text {eff }}\right)$ depends on the geometry of the samples as $E_{\text {eff }}=E+17 T l^{2} / 32 w t^{3}$. Thus, plotting of $E_{\text {eff }}$ vs $l^{2} / w$ for a fixed thickness (only single layers were selected), as illustrated in Figure 4b, yields a linear dependence from which an approximately constant value of $\sim 4 \mathrm{nN}$ for all the sheets can be inferred. Tensions introduced during the fabrication process represent a general hurdle in the development of nanoelectromechanical resonators. ${ }^{19,20}$ For the present samples, the tension is estimated to be a few nanonewtons, which is 2 orders of magnitude lower than that reported for mechanically exfoliated graphene. ${ }^{21-24}$ The presence of such large tensions in the latter case has been attributed to macroscopic uncontrolled forces applied
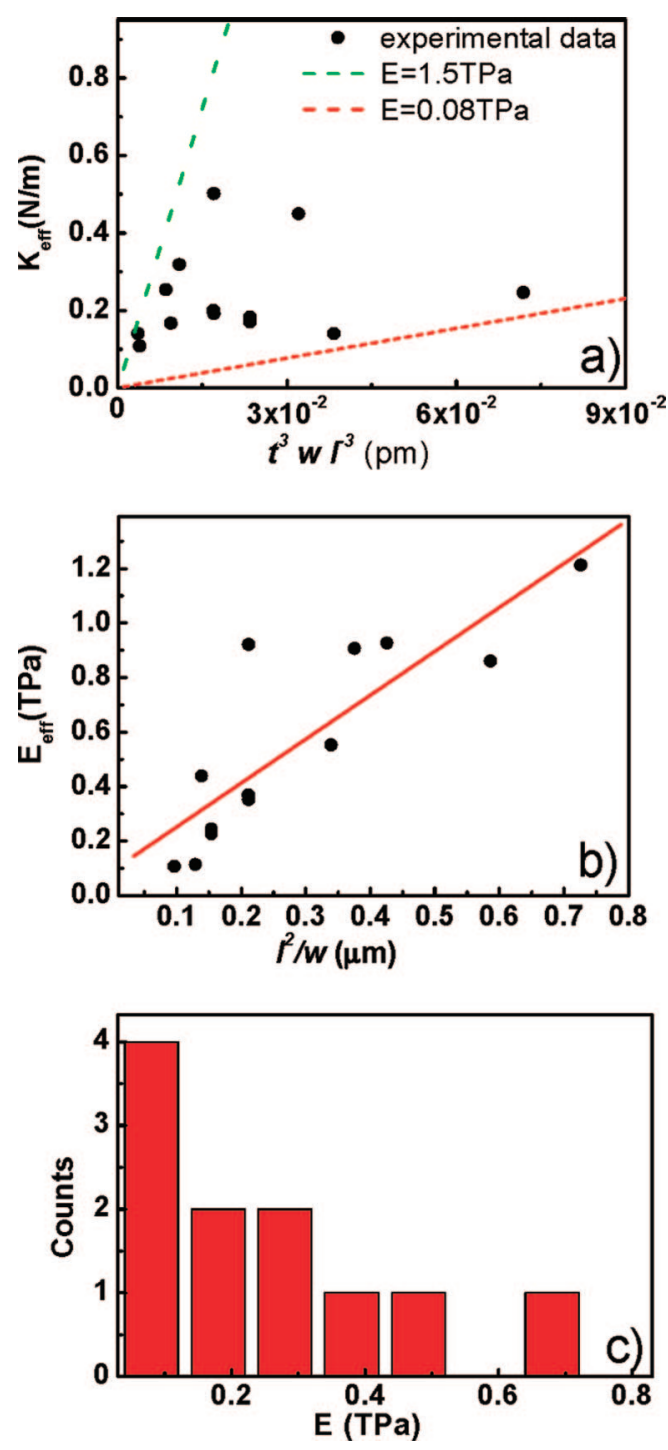

Figure 4. Elastic modulus for a range of reduced GO monolayers with different geometries. (a) Measured effective force constant vs $t^{3} w l^{-3}$ for different GO monolayer samples, with $t$ as the thickness, $w$ as width, and $l$ as the length of the sheets (note that $t$ $=1 \mathrm{~nm}$ is fixed since only monolayers were selected). The red and green dashed lines indicate the dependence of $K_{\text {eff }}$ expected for $E=1.8$ and $0.08 \mathrm{TPa}$, respectively (in the absence of built-in tension). (b) Plot of $E_{\text {eff }}$ as a function of $l / w$ for different monolayer samples. From the linear fit an averaged tension of $4 \mathrm{nN}$ can be extracted. (c) Histogram of the values of the elastic modulus obtained from the experimental data in panel a using eq 1 and the estimated value of $T$ from panel $b$.

during the deposition process. By contrast, the solution-based approach used in this work leads to significantly lower tensions, allowing easy access to the intrinsic properties of these ultrathin membranes.

According to eq 1 and based upon the determined tension value, a mean elastic modulus of $0.25 \mathrm{TPa}$ is calculated for the reduced GO monolayers, with a standard deviation of $0.15 \mathrm{TPa}$ (Figure 4c). The reproducibility of the results with different tips and the linear dependence observed in Figure $4 \mathrm{~b}$ (indication of experimental accuracy in determination of geometry and forces) suggest that the scattering present in the Young modulus is most likely to arise from a difference 

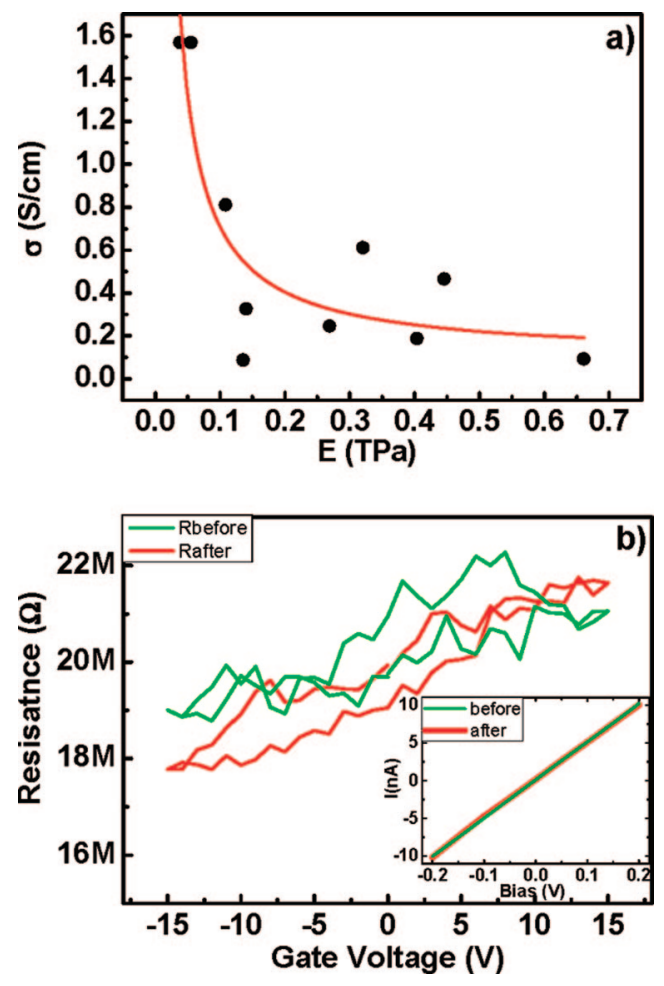

Figure 5. Correlation between electrical and mechanical properties. (a) Room temperature conductivity of different monolayers as a function of the elastic modulus. The experimental data have been fitted (red curve) by the expression $\sigma=A+B / E$ (with A, B fitting constants) as a guide to the eye. (b) Room temperature electrical resistance of the suspended GO layer shown in Figure 1, measured as a function of back gate voltage before (green curve) and after (red curve) repeated deformation by AFM tip indentation. The relatively weak gate dependence originates from the reduced capacitance between the layer and the conductive silicon substrate, due to the removal of the $\mathrm{SiO}_{2}$ dielectric. The inset shows corresponding $I-V$ curves recorded at zero gate potential before and after repeated indentations.

in microscopic structure of the sheets and not from experimental errors. The $E$ value of $0.25 \mathrm{TPa}$ is remarkably high for a $2 \mathrm{D}$ membrane and approaches that predicted for pristine graphene. ${ }^{25}$ At the same time, this value provides a boundary for the maximum mechanical performance that could be reached via optimization of the interlayer coupling within bulk, paper-like materials based on (reduced) GO. ${ }^{14}$

From Figure 5a, it can be seen that the conductivity of the reduced GO sheets scales inversely with the elastic modulus. On the basis of theoretical predictions of local bonding reinforcement, and consequently stiffening of the sheet by oxygen bridges introduced between the graphene carbons,${ }^{26}$ the sheets with higher elastic modulus and lower conductivity could be assigned to those of comparatively high oxygen content. However, unreduced samples with maximum oxygen content and a 2-3 orders of magnitude lower conductivity did not display a further enhanced elastic modulus (data not shown), which evidence a second factor governing the mechanical behavior. It is plausible to assume that this contribution arises from the contained structural defects, that is, holes or vacancies that constitute weak links limiting the overall elasticity of the sheets. The existence of such defects has been experimentally confirmed ${ }^{6}$ and predicted to significantly impart the mechanical strength of graphene. ${ }^{27}$ In contrast to the oxygen-containing functional groups, they cannot be removed upon chemical reduction and thus remain within the sheets.

A further reflection of the outstanding mechanical performance of the reduced GO sheets is the stability of their electrical properties against deformation. In fact, electrical measurements prior to and after repeated deformation showed that the resistance of the monolayers remained essentially unchanged, provided that the indentation depth did not exceed $10 \mathrm{~nm}$ (Figure 5b). The combined mechanical and electrical stability demonstrates that the electrical contacts, which are expected to be extremely sensitive against changes in the contact area and the GO-metal distance, are not affected during the deformation. For larger indentations $(\delta \approx 50 \mathrm{~nm})$, some devices exhibited a resistance increase by approximately $20 \%$, a finding that might be explained by a reduced physical contact between the GO sheet and the metal or the local shear strain introduced by the tip under these conditions.

Finally, it is noteworthy that reduced GO sheets comprised of more than three layers showed a markedly different behavior. In particular, they displayed 1 order of magnitude lower values of the elastic modulus, as compared to those of the single and double layers. A similar reduction of $E$ has been observed upon bundling of single-walled carbon nanotubes. ${ }^{28}$ This behavior is characteristic of stiffened structures and can be understood on the basis of competing bending and shear forces. For stiffer structures like the thicker and shorter multilayers, bending deflections are almost negligible and shear strains play a dominant role. Thus, their mechanical response is out of the bending dominated limit, such that the above performed analysis is not applicable anymore. Further support for the relevance of shear strain stems from the observation that the electrical resistance of the multilayers was consistently found to be significantly increased after the deformations.

In summary, our study of suspended, chemically derived graphene sheets reveals an elastic modulus closely approaching the value predicted for pristine graphene. Furthermore, the chemically reduced graphene oxide combines useful electrical conductivity with excellent mechanical properties including high bending flexibility and tensile strength. Due to their stability, robustness, and reversible response to mechanical stress, these ultrathin membranes represent promising candidates for application in nanoelectromechanical devices, for example, resonators with resonance frequencies in the microwave range. Moreover, their high robustness, low mass, and large surface area render such graphene-based membranes ideally suited as components of force, mass, or chemical sensors. Future experimental and theoretical studies addressing the influence of defects and oxygen functionalities contained in the graphene layers will be needed to achieve a balance of their mechanical and electrical properties.

Acknowledgment. We thank J. Weber for his help with the critical point drier and J. Gómez-Herrero for helpful discussions. C. Gómez-Navarro acknowledges support from the Alexander von Humboldt Foundation. 
Supporting Information Available: Detailed description of the sample preparation and experimental methods. This material is available free of charge via the Internet at http:// pubs.acs.org.

\section{References}

(1) Novoselov, K. S.; Geim, A. K.; Morozov, S. V.; Jiang, D.; Zhang, Y.; Dubonos, S. V.; Grigorieva, I. V.; Firsov, A. A. Science 2004, 306 (5696), 666-669.

(2) Novoselov, K. S.; Jiang, D.; Schedin, F.; Booth, T. J.; Khotkevich, V. V.; Morozov, S. V.; Geim, A. K. Proc. Natl. Acad. Sci. U.S.A. 2005, 102 (30), 10451-10453.

(3) Berger, C.; Song, Z. M.; Li, X. B.; Wu, X. S.; Brown, N.; Naud, C.; Mayo, D.; Li, T. B.; Hass, J.; Marchenkov, A. N.; Conrad, E. H.; First, P. N.; de Heer, W. A. Science 2006, 312 (5777), 1191-1196.

(4) Ohta, T.; Bostwick, A.; Seyller, T.; Horn, K.; Rotenberg, E. Science 2006, 313 (5789), 951-954.

(5) Stankovich, S.; Piner, R. D.; Chen, X. Q.; Wu, N. Q.; Nguyen, S. T.; Ruoff, R. S. J. Mater. Chem. 2006, 16 (2), 155-158.

(6) Gomez-Navarro, C.; Weitz, R. T.; Bittner, A. M.; Scolari, M.; Mews, A.; Burghard, M.; Kern, K. Nano Lett. 2007, 7 (11), 3499-3503.

(7) Gilje, S.; Han, S.; Minsheng, W.; Kang, L. W.; Kaner, R. B. Nano Lett. 2007, 7 (11), 3394-3398.

(8) Li, D.; Muller, M. B.; Gilje, S.; Kaner, R. B.; Wallace, G. G. Nat. Nanotechnol. 2008, 3 (2), 101-105.

(9) Lerf, A.; He, H. Y.; Forster, M.; Klinowski, J. J. Phys. Chem. B 1998, 102 (23), 4477-4482.

(10) Li, J. L.; Kudin, K. N.; McAllister, M. J.; Prud'homme, R. K.; Aksay, I. A.; Car, R. Phys. Rev. Lett. 2006, 96 (17),

(11) Schniepp, H. C.; Li, J. L.; McAllister, M. J.; Sai, H.; Herrera-Alonso, M.; Adamson, D. H.; Prud'homme, R. K.; Car, R.; Saville, D. A.; Aksay, I. A. J. Phys. Chem. B 2006, 110 (17), 8535-8539.
(12) Stankovich, S.; Dikin, D. A.; Dommett, G. H. B.; Kohlhaas, K. M.; Zimney, E. J.; Stach, E. A.; Piner, R. D.; Nguyen, S. T.; Ruoff, R. S. Nature 2006, 442 (7100), 282-286.

(13) Kotov, N. A.; Dekany, I.; Fendler, J. H. Adv. Mater. 1996, 8 (8), 637.

(14) Dikin, D. A.; Stankovich, S.; Zimney, E. J.; Piner, R. D.; Dommett, G. H. B.; Evmenenko, G.; Nguyen, S. T.; Ruoff, R. S. Nature 2007, 44 (7152), 457-460.

(15) Hummers, W. S.; Offeman, R. E. J. Am. Chem. Soc. 1958, 80 (6), 1339-1339.

(16) Gere, J. M. Mechanics of materials; Thomson: Andover, U.K., 2006.

(17) Wan, K. T.; Guo, S.; Dillard, D. A. Thin Solid Films 2003, 425 (12), 150-162.

(18) San Paulo, A.; Bokor, J.; Howe, R. T.; He, R.; Yang, P.; Gao, D.; Carraro, C.; Maboudian, R. Appl. Phys. Lett. 2005, 87 (5),

(19) Ekinci, K. L.; Roukes, M. L. Rev. Sci. Instrum. 2005, 76 (6),

(20) Mueggenburg, K. E.; Lin, X. M.; Goldsmith, R. H.; Jaeger, H. M. Nat. Mater. 2007, 6 (9), 656-660.

(21) Frank, I. W.; Tanenbaum, D. M.; Van der Zande, A. M.; McEuen, P. L. J. Vac. Sci. Technol., B 2007, 25 (6), 2558-2561.

(22) Garcia-Sanchez, D.; van der Zande, A. M.; Paulo, A. S.; Lassagne, B.; McEuen, P. L.; Bachtold, A. Nano Lett. 2008, 8, 1399-1403.

(23) Poot, M.; van der Zant, H. S. J. Appl. Phys. Lett. 2008, 92 (6),

(24) Bunch, J. S.; van der Zande, A. M.; Verbridge, S. S.; Frank, I. W.; Tanenbaum, D. M.; Parpia, J. M.; Craighead, H. G.; McEuen, P. L. Science 2007, 315 (5811), 490-493.

(25) Sanchez-Portal, D.; Artacho, E.; Soler, J. M.; Rubio, A.; Ordejon, P. Phys. Rev. B 1999, 59 (19), 12678-12688.

(26) Incze, A.; Pasturel, A.; Peyla, P. Phys. Rev. B 2004, 70 (21),

(27) Paci, J. T.; Belytschko, T.; Schatz, G. C. J. Phys. Chem. C 2007, 111 (49), 18099-18111.

(28) Salvetat, J. P.; Briggs, G. A. D.; Bonard, J. M.; Bacsa, R. R.; Kulik, A. J.; Stockli, T.; Burnham, N. A.; Forro, L. Phys. Rev. Lett. 1999, 82 (5), 944-947.

NL801384Y 